\title{
Combined adductor canal and i-PAK blocks is better than combined adductor canal and periarticular injection blocks for painless $A C L$ reconstruction surgery
}

\begin{abstract}
The target of the present study is dual. First, to test the hypothesis of combined nerve block addressing different sites of nerve course can result in more successful distribution and prolongation of block whenever lower concentration local anesthetic mixture is used. Secondly to compare effectiveness of combined Adductor Canal and i-PAK Blocks versus Combined Adductor Canal and Periarticular Injection Blocks for ameliorating postoperative ACL Reconstruction Surgery pain.
\end{abstract}

Material and methods: The study is a Prospective, randomized cohort study: After IRB (Institutional Review Board) and committee approval was obtained, Patients aged 18-75years undergoing ACL Reconstruction Surgery over the period of one year and included 246 patients which were randomly allocated to either Combined Adductor Canal and i-PAK Blocks (group A), or Combined Adductor Canal and Periarticular Injection Blocks (group B) groups, each had 123 patients at the end of the study. Informed consent for this study was taken from the patient. This is a scientific abstract with no patient protected health information. A standard local anesthetic mixture was used throughout the study. The mixture of Ropivacaine $3.75 \%$ with $4 \mathrm{mg}$ of dexamethasone in a total volume of $42 \mathrm{ml}$ of mixture. There is no off-label medications included. No promotional content of a commercial entity is included (brand/trade/product names, photos, logos, company names, etc.).

Results: This study of the U/S guided comparison of combined Adductor Canal and i-PAK Blocks versus Combined Adductor Canal and Periarticular Injection Blocks For ACL Reconstruction Surgery demonstrates that of combined Adductor Canal and i-PAK Blocks results in prolongation of the time before request for first analgesia rescue (h) (statistically significant) indicated by higher readings in group A compared to group B .

Statistically significant difference was also observed comparing the two groups concerning the total pethidine consumption $(\mathrm{mg})$ in the first 48 post-operative hours with much reduction of the total dose in group A together compared to group B.

Postoperative VAPS at rest and on walking were reduced after of combined Adductor Canal and i-PAK Blocks at all-time points assessed compared to group B.
Volume 10 Issue 4 - 2018

\author{
Nagi Amer \\ Professor at Cairo University, UAE
}

Correspondence: Nagi Amer, Professor at Cairo University,VILLA 90 NARGIS 4, United Arab Emirates, Tel 00971508422635, Email naggiaam@hotmail.com

Received: July 20, 2018 | Published: August 14, 2018

Discussion: The combination of distal blocks is having the advantage of being safer approach away from critical structures and allows preservation of proximal muscle function. The inability to move due to the motor block of proximal and distal musculature has been shown to decrease patient satisfaction. Distal peripheral nerve blocks are superior at preserving motor function of the operative limb. A recent randomized controlled trial addressing this subject in the upper extremity (comparing ultrasound guided supraclavicular plexus block with distal peripheral nerve blocks for outpatient hand surgery) Showed better strength preservation and greater patient satisfaction with distal blocks. Using multiple points of blocking the nerves to reach the sensory terminals supplying the knee region with motor sparing effect can be achieved using lower anesthetic concentrations. Moreover, combined nerve block addressing different sites of nerve course targeting those which are mainly sensory can result in more successful analgesia and sparing of motor branches of mixed nerves. The validation of the present study results essentially requires further large RCT of adequate sample size.

Conclusion: This study reiterates the importance of tackling multimodal approach in regional techniques which probably will have a place in tailoring patient and surgical pain relief needs. The best approach should be tailored to suit specific surgery whenever possible. Combined Adductor Canal and i-PAK Blocks is better than Combined Adductor Canal and Periarticular Injection Blocks for ACL Reconstruction Surgery concerning postoperative pain.
Abbreviations: ACL, anterior cruciate ligament; FNB, femoral nerve block; ACB, adductor canal block

\section{Introduction}

The dream of painless surgery is more and truer whenever skills and knowledge of managing pain pathways is getting increased. Moreover postoperative analgesia is an utmost importance for successful surgical outcome.

Targeting postoperative pain relief in major knee surgery is a real challenge for the new advancements in technology and knowledge.
The development of ultrasound guidance to block acute and chronic pain has gained more and more trust in the last decade. Among some of the most commonly used techniques for major knee surgery pain postoperatively are Adductor Canal and i-PAK Blocks as well as Periarticular Injection, all could be used as the main component of multimodal analgesia postoperatively.

By blocking the distal branches of the femoral nerve in the midthigh, the adductor canal block (ACB) can spare quadriceps muscle strength while providing analgesia similar to an ordinary femoral nerve block (FNB) for in patients undergoing major knee surgery. 
ANTERIOR cruciate ligament (ACL) reconstruction is one of the most commonly performed orthopedic procedures. ${ }^{1}$ The capability to perform ACL reconstruction on outpatient basis is mainly dependent on an effective analgesic strategy that minimizes the role of systemic analgesics yet provides adequate postoperative pain control and eliminates the need for overnight stay or readmission. ${ }^{2}$

The adductor canal contains the vastus medialis nerve, medial femoral cutaneous nerve, articular branches from the obturator nerve, and the medial retinacular nerve in addition to saphenous nerve that innervate the medial, lateral, and anterior aspects of the knee. ${ }^{3,4}$ Theoretically, ACB is almost a pure sensory nerve block, which only affects the motor function of vastus medialis due to its impact on nerve to the muscle as it crosses the adductor canal ${ }^{5}$ and decrease the incidence of fall. ${ }^{6}$ Moreover, multiple clinical studies and several meta-analyses ${ }^{7-9}$ have suggested that ACB could provide quadriceps muscle strength preservation, better ambulatory ability, and earlier functional recovery with comparable analgesic effects and morphine consumption when compared to FNB in patients after TKA.

Understanding of adductor canal anatomy, including nerves related to ACL reconstruction, is still not very clear which at least precludes theoretical assumption of analgesic effectiveness of the ACB. ${ }^{10}$ For example, descriptions of the anatomical communication between the saphenous and medial femoral cutaneous nerves in the adductor canal have been inconsistent. ${ }^{10,11}$

Furthermore, the posterior branch of the medial femoral cutaneous nerve, which gives cutaneous innervation to the medial thigh and leg, frequently do not pass in the adductor canal in up to $40 \%$ of cases, while its anterior branch, which provides cutaneous innervation to the medial and lateral knee, is never part of the adductor canal. $3,10,11$

Moreover, patients who undergo TKA and receive an FNC block or ACB frequently complain of postoperative posterior knee pain needing supplemental narcotic medications. ${ }^{12-14}$ The sciatic nerve block has been shown to improve analgesia and reduce narcotic consumption when combined with the FNC block. ${ }^{15}$ However, a sciatic nerve block can result in sensory and motor deficits below the knee and may increase the danger of falls. ${ }^{16}$ Therefore, a better regional anesthetic technique would include a regional block that provides analgesia to the posterior knee but does not cause distal neurologic weakness.

A novel nerve block technique described by Sanjay Sinha, MD, from Hartford, CT, is an ultrasound (US)-guided local anesthetic injection between the popliteal artery and the capsule of the knee (IPACK). ${ }^{17}$ By targeting only the terminal branches of the sciatic nerve, the IPACK block provides an alternative for decreasing posterior knee pain following TKA and greatly avoid foot drop. ${ }^{18}$ However, clinical evidence comparing the effectiveness of analgesia, postoperative physical rehabilitation, and opioids reduction with the addition of an IPACK block to ACB is limited. The objective of this study was to evaluate the effect of an IPACK block supplementing ACB block on postoperative pain scale scores, opioid consumption, performance during early physical therapy, and duration of hospital length of stay in patients undergoing unilateral ACL repair.

\section{Methods}

The study is a Prospective, randomized cohort study: Patients aged 18-75years undergoing ACL Reconstruction Surgery over the period of one year and included 246 patients which were randomly allocated to either Combined Adductor Canal and i-PAK Blocks (group A), or Combined Adductor Canal and Periarticular Injection Blocks (group B) groups, each had 123 patients at the end of the study. Informed consent for this study was taken from the patient. This is a scientific abstract with no patient protected health information..$^{20-22}$

A standard local anesthetic mixture was used throughout the study. The mixture of Ropivacaine $3.75 \%$ with $4 \mathrm{mg}$ of dexamethasone in a total volume of $42 \mathrm{ml}$ of mixture. Exclusion criteria included contraindication for regional anesthetic, chronic narcotic use, allergies to local anesthetics, intraoperative use of inhalational anesthetics, previous neuropathy on the operative limb, ages younger than 18 or older than $75 \mathrm{yr}$, and ASA class 4 or 5 . In the holding area, patients were sedated with intravenously administered midazolam before performance of the nerve block and after giving spinal anesthesia, with $2.5 \mathrm{cc}$ of $0.5 \%$ bupivacaine as the spinal agent. Pprophylactic administration of dexamethasone and ondansetron to reduce PONV.

\section{Group A}

An ultrasound-guided ACB (15cc of local anesthetic mixture via a 22 -gauge $8 \mathrm{~cm}$ sonoplex needle; was performed at mid-thigh level using a high- frequency transducer 5-12 $\mathrm{MHz}$ (Zonare ultrasound system before the surgery In supine position -and using the inplane technique with a 22 -gauge Sonoplex needle and $15 \mathrm{ml}$ of local anesthetic mixture was injected and the drug spread in the plane was observed. followed by $20 \mathrm{cc}$ ifilteration of the same anesthetic mixture injection between the popliteal artery and the capsule of the knee (IPACK) using curved array transducer C6-2 (Zonare ultrasound system). In supine position with the knee slightly flexed and externally rotated.

\section{Group B}

An ultrasound-guided ACB (15cc of via a 22 -gauge $8 \mathrm{~cm}$ sonoplex needle; was performed at mid-thigh level using a high- frequency transducer 5-12 MHz (Zonare ultrasound system) before the surgery In supine position -and using the in-plane technique with a 22-gauge Sonoplex needle and $15 \mathrm{ml}$ of local anesthetic mixture was injected and the drug spread in the plane was observed. $20 \mathrm{cc}$ infiltration of the same anesthetic mixture infiltrated in the knee during the operation, and 2 bolus injections of the same mixture were given via an intraarticular catheter postoperatively.

In operation theatre non-invasive monitoring (ECG, Oximeter, Noninvasive Blood Pressure) were attached.

Intraoperative data recorded the total time to perform the block (starting from needle insertion to exit), surgery time, tourniquet pressure, and time.

Quadriceps motor weakness and sensory exam (leg pinprick and temperature discrimination) was assessed for legs at 6 to 8,24 , and $48 \mathrm{~h}$ after anesthesia recovery. Block success was verified by testing for pinprick sensation in the distribution of saphenous nerve as well as regainig adequate motor function. The primary outcome was measured by evaluated by the patient's ability to actively extend the knee while flexed at 45 degrees.

Additional analgesia was provided by injection paracetamol $1 \mathrm{G}$ intravenous 8 hrly for first 72 hours and parecoxib sodium $40 \mathrm{mg}$ iv 12 hourly . Injection pethidine $50 \mathrm{mg}$ when required was advised for rescue analgesia. Pain scores using Visual Analogue Scale (VAS) were monitored two hourly for 24 hours and then 4 hrly for next 24 hours. 


\section{Results}

Our study of the U/S guided comparing Combined Adductor Canal and i-PAK Blocks, versus Combined Adductor Canal and Periarticular Injection Blocks show no statistically significant difference concerning the demographic data. On the other hand time before request for first analgesia rescue (h) (statistically significant) indicated by higher readings in group A $(32.35 \pm 3.20 \mathrm{~h})$ compared to group B (21.40 \pm 2.82$)$. Statistically significant difference was also observed comparing the two groups concerning the total pethidine consumption (mg) in the first 48 post-operative hours (Table 1).

Postoperative VAPS at rest were reduced after of Combined Adductor Canal and i-PAK Blocks, versus Combined Adductor Canal and Periarticular Injection Blocks at all time points assessed (Table 1).

Table I Postoperative pethidine requirements and VAPS (mean \pm SD)

\begin{tabular}{llll}
\hline Group & Group A & Group B & P value \\
$\begin{array}{l}\text { Request for first analgesia } \\
\text { rescue (h) }\end{array}$ & $32.35 \pm 3.20$ & $21.40 \pm 2.82$ & $<0.00$ I \\
$\begin{array}{l}\text { Total pethidine consumption } \\
\text { (mg) }\end{array}$ & $28.13 \pm 33.2 \mathrm{I}$ & $6 \mathrm{I} .33 \pm 73.63$ & $<0.00 \mathrm{I}$ \\
$\begin{array}{l}\text { Postoperative visual analogue } \\
\text { pain score (VPAS) 4hours }\end{array}$ & $0(0-0.25)$ & $\mathrm{I} .5(\mathrm{I} .5-2.25)$ & $0.00 \mathrm{I}$ \\
$\begin{array}{l}\text { Postoperative visual analogue } \\
\text { pain score (VPAS) 8hours }\end{array}$ & $0(0-0.25)$ & $\mathrm{I} .5(\mathrm{I} .5-2.25)$ & $0.00 \mathrm{I}$ \\
$\begin{array}{l}\text { Postoperative visual analogue } \\
\text { pain score (VPAS) I6hours }\end{array}$ & $0(0-0.25)$ & $\mathrm{I} .5(\mathrm{I} .5-2.25)$ & $<0.00 \mathrm{I}$ \\
$\begin{array}{l}\text { Postoperative visual analogue } \\
\text { pain score (VPAS) 24hours }\end{array}$ & $0(0-0.25)$ & $6(6-7)$ & $<0.00 \mathrm{I}$ \\
$\begin{array}{l}\text { Postoperative visual analogue } \\
\text { pain score (VPAS) 48hours }\end{array}$ & $0(0-0.25)$ & $6(6-7)$ & $<0.00 \mathrm{I}$ \\
\hline
\end{tabular}

\section{Discussion}

Acute postoperative pain is a common problem encountered by all medical professionals as per evidence based practice guidelines. As a part of the accepted spectrum of perioperative anesthetic care, anesthetists play a major role where acute postoperative pain control affects surgical outcomes that may cause prolonged hospital stays and patient dissatisfaction. Among the most frequently performed surgeries ACL Reconstruction Surgery occupy a major part of every day and day care practice.

The target of the present study was dual. First, to test the hypothesis of combined nerve block addressing different sites of nerve course targeting those which are mainly sensory can result in more successful analgesia and sparing of motor branches of mixed nerves particularly, whenever lower concentration local anesthetic mixture is used. Secondly to compare effectiveness of combined Adductor Canal and i-PAK Blocks versus Combined Adductor Canal and Periarticular Injection Blocks for ACL Reconstruction Surgery. Several studies validated motor sparing quality of analgesia. Distal approaches to upper extremity block with patient satisfaction are a good example. A recent randomized controlled trial comparing ultrasound guided supraclavicular plexus block with distal peripheral nerve blocks for outpatient hand surgery Showed better strength preservation and greater patient satisfaction with distal blocks. (Nicholas Lam, Anesthesiology Research and Practice 2014)

However large dose of local anesthetic during adductor Canal Block could result in quadriceps weakness from proximal spread. Therefore the current study used a lower dose of anesthetic concentration as well as volume to achieve this goal.

A major concern among practitioners is whether an $\mathrm{ACB}$ provides enough sensory coverage for different knee surgeries particularly TKA. It is important to note that the ACB performed at the level of the mid-thigh involves not only the saphenous nerve but several other sensory nerves that innervate the medial, lateral, and anterior aspects of the knee, encompassing the superior pole of the patella to the proximal tibia.

Opioid consumption was different in both groups. Highly significant difference was also observed comparing the two groups concerning the total pethidine consumption $(\mathrm{mg})$ in the first 48 postoperative hours (Table 1). The occurrence of postoperative nausea and vomiting was low in both groups with no statistical difference. This is most likely due to the prophylactic administration of dexamethasone and ondansetron given routinely to all patients, as well as the avoidance of intraoperative narcotics and volatile agents.

On the whole, combined Adductor Canal and i-PAK Blocks was better than Combined Adductor Canal and Periarticular Injection Blocks for ACL Reconstruction Surgery concerning postoperative pain. The addition of i-PAK had higher analgesia effect than the addition of Periarticular injection might be attributed to the better distribution of the first injection to reach the terminal sensory braches both inside and outside the knee capsule when using lower anesthetic concentrations.

he use of dividing the dose proved to be a good strategy to get maximum analgesia. The validation of the present study results essentially requires further large RCT of adequate sample size. The best approach needs further validation and should be tailored to suit specific surgery whenever possible to facilitate future study planning, utilize the most updated knowledge, and support the advances of techniques in regional blocks.

\section{Conclusion}

This study reiterates the importance of tackling multimodal approach in regional techniques which probably will have a place in tailoring patient and surgical pain relief needs. The best approach should be tailored to suit specific surgery whenever possible. Combined Adductor Canal and i-PAK Blocks is better than Combined Adductor Canal and Periarticular Injection Blocks for ACL Reconstruction Surgery concerning postoperative pain.

\section{Consent}

Informed consent for this study was taken from the patient.

\section{Acknowledgements}

None.

\section{Conflict of interest}

Author declares there is no conflict of interest towards this article. 


\section{References}

1. Bach BR. Revision anterior cruciate ligament surgery. Arthroscopy. 2003;19(Suppl 1):14-29.

2. Tierney GS, Wright RW, Smith JP. DA Anterior cruciate ligament reconstruction as an outpatient procedure. Am J Sports Med. (1995);23(6):755-756.

3. Horner G, Dellon AL. Innervation of the human knee joint and implications for surgery. Clin Orthop. 1994;(301):221-226.

4. Bushnell BD, Sakryd G, Noonan TJ. Hamstring donor-site block: Evaluation of pain control after anterior cruciate ligament reconstruction. Arthroscopy. 2010;26(7):894-900.

5. Shah NA, Jain NP. Is continuous adductor canal block better than continuous femoral nerve block after total knee arthroplasty? Effect on ambulation ability, early functional recovery and pain control: a randomized controlled trial. J Arthroplasty. 2014;29(11):2224-2229.

6. Memtsoudis SG, Yoo D, Stundner O, et al. Subsartorial adductor canal vs femoral nerve block for analgesia after total knee replacement. Int Orthop. 2015;39(4):673-680.

7. Gao F, Ma J, Sun W, et al. Adductor Canal Block Versus Femoral Nerve Block for Analgesia After Total Knee Arthroplasty: A Systematic Review and Meta-analysis. Clin J Pain. 2016;33(4):356-368.

8. Li D, Ma GG. Analgesic efficacy and quadriceps strength of adductor canal block versus femoral nerve block following total knee arthroplasty. Knee Surg Sports Traumatol Arthrosc. 2016;24(8):2614-2619.

9. Kerr DR, Kohan L. Local infiltration analgesia: a technique for the control of acute postoperative pain following knee and hip surgery: a case study of 325 patients. Acta Orthop. 2008;79(2):174-183.

10. Mochizuki T, Akita K, Muneta T, et al. Anatomical bases for minimizing sensory disturbance after arthroscopically-assisted anterior cruciate ligament reconstruction using medial hamstring tendons. Surg Radiol Anat. 2003;25(3-4):192-199.

11. Oh SJ, Hatanaka Y, Ohira M, et al. Clinical utility of sensory nerve conduction of medial femoral cutaneous nerve. Muscle Nerve. 2012;45(2):195-199.
12. Yadeau JT, Goytizolo EA, Padgett DE, et al. Analgesia after total knee replacement: local infiltration versus epidural combined with a femoral nerve blockade: a prospective, randomized pragmatic trial. Bone Joint J. 2013; 95-B(5):629-635.

13. Sinha SK, Abrams JH, Arumugam S, et al. Femoral nerve block with selective tibial nerve block provides effective analgesia without foot drop after total knee arthroplasty: a prospective, randomized, observer-blinded study. Anesth Analg. 2012;115(1):202-206.

14. Vendittoli PA, Makinen P, Drolet P, et al. A multimodal analgesia protocol for total knee arthroplasty: a randomized, controlled study. J Bone Joint Surg Am. 2006;88(2):282-289.

15. Pham Dang C, Gautheron E, Guilley J, et al. The value of adding sciatic block to continuous femoral block for analgesia after total knee replacement. Reg Anesth Pain Med. 2005; 30(2):128-133.

16. Baratta JL, Gandhi K, Viscusi ER. Perioperative pain management for total knee arthroplasty. J Surg Orthop Adv. 2014; 23(1):22-36.

17. Thobhani S, Scalercio L, Elliott CE, et al. Novel Regional Techniques for Total Knee Arthroplasty Promote Reduced Hospital Length of Stay: An Analysis of 106 Patients. Ochsner J. 2017;17(3):233-238.

18. https://issuu.com/mcmahongroup/docs/mman0012_2012_tab.

19. Atkinson HD, Hamid I, Gupte CM, et al. Postoperative fall after the use of the 3-in-1 femoral nerve block for knee surgery: A report of four cases. $J$ Orthop Surg (Hong Kong). 2008;16(3):381-384.

20. Busch CA, Shore BJ, Bhandari R, et al. Efficacy of periarticular multimodal drug injection in total knee arthroplasty. A randomized trial. $J$ Bone Joint Surg Am. 2006;88(5):959-963.

21. Allen HW, Liu SS, Ware PD, et al. Peripheral nerve blocks improve analgesia after total knee replacement surgery. Anesth Analg. 1998;87(1):93-97.

22. Lam NC, Charles M, Mercer D, et al. A Triple-Masked, Randomized Controlled Trial Comparing Ultrasound-Guided Brachial Plexus and Distal Peripheral Nerve Block Anesthesia for Outpatient Hand Surgery. Anesthesiol Res Pract. 2014;2014:324083. 